\title{
Shikonin suppresses proliferation and induces apoptosis in human leukemia NB4 cells through modulation of MAPKs and c-Myc
}

\author{
ZHI-LING SHAN ${ }^{1,2}$, LIANG ZHONG ${ }^{2}$, CHU-LAN XIAO $^{1}$, LIU-GEN GAN ${ }^{1}$, \\ TING XU ${ }^{1}$, HAO SONG ${ }^{1}$, RONG YANG ${ }^{2}$, LIU LI $^{2}$ and BEI-ZHONG LIU ${ }^{1,2}$ \\ ${ }^{1}$ Central Laboratory of Yongchuan Hospital, Chongqing Medical University, Chongqing 402160; \\ ${ }^{2}$ Key Laboratory of Laboratory Medical Diagnostics, Ministry of Education, Department of \\ Laboratory Medicine, Chongqing Medical University, Chongqing 400016, P.R. China
}

Received September 10, 2016; Accepted May 18, 2017

DOI: $10.3892 / \mathrm{mmr} .2017 .6965$

\begin{abstract}
Acute promyelocytic leukemia (APL) is a special subtype of acute myeloid leukemia that responds to treatment with all-trans retinoic acid and arsenic trioxide. However, severe side effects and drug resistance limit the effectiveness of these treatments. Hence, new drugs for APL are required urgently. Shikonin, an active naphthoquinone derived from the Chinese medical herb Zi Cao exerts antitumor activity in several cancers. In the present study, the effects of shikonin on proliferation and apoptosis in NB4 cells, as well as related mechanisms were assessed. Treatment of NB4 cells with shikonin inhibited proliferation in a concentration- and time-dependent manner. The cell cycle was arrested in the G1 phase. NB4 cells treated with shikonin exhibited more apoptosis and higher levels of cleaved caspase-3 and poly ADP-ribose polymerase than control cells. Western blotting results demonstrated that the expression of p-p38 mitogen-activated protein kinase (p-p38MAPK) and p-c-Jun N-terminal kinase (p-JNK) was increased significantly by shikonin treatment, while the expression of p-ERK and c-Myc was decreased. In summary, these findings indicated that shikonin inhibited cell proliferation and induced apoptosis partly through modulation of the MAPKs and downregulation of c-Myc.
\end{abstract}

\section{Introduction}

Acute promyelocytic leukemia (APL) is a subtype of acute myeloid leukemia accounting for 5-15\% of all forms of this disease (1). APL is characterized by a chromosomal translocation $[\mathrm{t}(15 ; 17)(\mathrm{q} 24 ; \mathrm{q} 21)]$ resulting in the formation of a retinoic acid receptor $\alpha$ and promyelocytic leukemia (PML-RAR $\alpha$ )

Correspondence to: Dr Bei-Zhong Liu, Central Laboratory of Yongchuan Hospital, Chongqing Medical University, 439 Xuanhua Road, Chongqing 402160, P.R. China

E-mail: liubeizhong@cqmu.edu.cn

Key words: shikonin, proliferation, apoptosis, leukemia NB4 cells, mitogen-associated protein kinases, c-Myc fusion protein (1-3). All-trans retinoic acid (ATRA) and arsenic trioxide (ATO) are the most important treatments of APL patients and induce a high cure rate. ATRA promotes differentiation and ATO induces apoptosis. Although ATRA and ATO have made APL highly curable, some patients may develop severe side effects. In addition, some APL patients are not sensitive to these compounds $(1,4-7)$. Hence, it is necessary to develop new therapeutic strategies for this disease.

Shikonin, an active component of the Chinese medical herb $\mathrm{Zi} \mathrm{Cao} \mathrm{has} \mathrm{been} \mathrm{used} \mathrm{to} \mathrm{treat} \mathrm{burns,} \mathrm{carbuncles,} \mathrm{macular}$ eruptions, measles and sore throats $(8,9)$. In addition to the anti-bacterial and anti-inflammatory activities of this medicine, shikonin inhibits proliferation and induces apoptosis in different cancer cell lines including prostate cancer (10), oral squamous cell carcinoma (11), chronic myelogenous leukemia (12), hepatocellular carcinoma (13) and thyroid cancer (14). Substantial evidence indicates that shikonin induces apoptosis partly through the mitogen-activated protein kinase (MAPK) pathway $(12,15)$.

The MAPK family, that includes extracellular signal-regulated kinase (ERK), p38 MAPK and c-Jun N-terminal kinase (JNK), serves an important role in cell proliferation, cell cycle progression, differentiation, survival and apoptosis $(16,17)$. The ERK pathway is primarily activated by growth factors and mitogens, and is important in cell growth and differentiation (18). p38 MAPK and JNK are mainly responsive to stress signals and inflammatory cytokines, and are associated with apoptosis $(19,20)$.

Accumulating evidence indicates that shikonin exerts antitumor activity in different cancer cells. However, the effects and related mechanism of shikonin on human leukemia NB4 cells are not known. In the present study, the authors investigated the influence of shikonin on the proliferation and apoptosis of NB4 cells and explored the potential mechanisms. The results may be beneficial for developing improved therapies for APL.

\section{Materials and methods}

Reagents. Shikonin and dimethylsulfoxide were purchased from Sigma-Aldrich; Merck KGaA, Darmstadt, Germany). The purity of shikonin was $>98 \%$. Antibodies against caspase-3, poly ADP-ribose polymerase (PARP), c-Myc, p-ERK1/2, 
ERK1/2, p38 MAPK, p-JNK and JNK were purchased from Cell Signaling Technology, Inc. (Danvers, MA, USA). The antibody against p-p38 MAPK was purchased from Merck KGaA. Goat anti-rabbit, goat anti-mouse and $\beta$-actin antibodies were purchased from Beijing Zhongshan Golden Bridge Biotechnology Co., Ltd. (Beijing, China).

Cell lines and culture. NB4 cells were obtained from the Shanghai Institute for Biological Science, Chinese Academy of Sciences (Shanghai, China) and maintained at $37^{\circ} \mathrm{C}$ under 5\% $\mathrm{CO}_{2}$ in RPMI-1640 (Gibco; Thermo Fisher Scientific, Inc., Waltham, MA, USA) medium containing $10 \%$ fetal calf serum (Gibco; Thermo Fisher Scientific, Inc.).

Cell proliferation assays. Cell viability was detected by the Cell Counting Kit (CCK)-8 (Sevenseas Futai Biotechnology Co., Ltd., Shanghai, China) assay. Cells $\left(1 \times 10^{4}\right)$ were seeded in 96-well plates and treated with increasing concentrations of shikonin for 12,24 and $36 \mathrm{~h}$. A total of $10 \mu \mathrm{l} \mathrm{CCK-8} \mathrm{solution}$ was added to each well at the end of each culture period. Cells were then incubated for $2 \mathrm{~h}$ at $37^{\circ} \mathrm{C}$ and absorbance of the medium was measured at $450 \mathrm{~nm}$ using a spectrophotometer.

Nucleus morphological changes examined by Hochest 33342 staining. Cells $\left(5 \times 10^{5}\right)$ were seeded in six-well plates and treated with shikonin $(0,0.3 \mu \mathrm{mol} / \mathrm{l})$ for $24 \mathrm{~h}$. Then, cells were washed with PBS three times, fixed with cold methanol overnight. Cells were washed with PBS three further times and stained with Hoechst 33342 (Beyotime Institute of Biotechnology, Beijing, China) for $5 \mathrm{~min}$ in the dark. Following three washes, the cells were observed using fluorescence microscopy.

Flow cytometry analysis. NB4 cells were treated with $0.3 \mu \mathrm{mol} / 1$ shikonin for $24 \mathrm{~h}$. Treated and control cells were harvested by centrifugation at $1,000 \mathrm{x}$ g for $5 \mathrm{~min}$ then washed and re-suspended with cold PBS. Cells were then stained with Annexin $\mathrm{V}$ and propidium iodide for 5-15 min at room temperature using the Annexin V/PI Apoptosis Detection kit (KeyGene, Wageningen, The Netherlands). Apoptosis was analyzed on a flow cytometer (BD Biosciences, Franklin Lakes, NJ, USA). The experiment was repeated at least three times.

The effects of shikonin on cell cycle distribution were detected with a cell cycle kit according to the manufacturer's instructions. Cells treated with $0.3 \mu \mathrm{mol} / 1$ shikonin for $24 \mathrm{~h}$ and control cells were collected by centrifugation, followed by washing, fixation and propidium iodide staining. The cell cycle distribution was examined by flow cytometry (BD Biosciences).

Western blot analyses. Cells were lysed with cold radioimmunoprecipitation assay lysis buffer (Beyotime Institute of Biotechnology) containing protease inhibitor cocktail for $10 \mathrm{~min}$. Cell proteins were collected by centrifuging at $13,000 \mathrm{x} g$ for $30 \mathrm{~min}$ and used for immunoblotting analyses. Cell proteins $(60 \mu \mathrm{g})$ were separated by SDS-PAGE and transferred to polyvinylidene fluoride membranes (EMD Millipore, Billerica, MA, USA). The membranes were blocked with 5\% non-fat milk dissolved in TBS with 20\% Tween-20, and then incubated with primary antibodies against caspase-3 (no. 9665; 1:1,000), PARP (no. 9532; 1:1,000), c-Myc (no. 5605; 1:1,000), p-ERK1/2 (no. 4370; 1:1,000), ERK1/2 (no. 4695; 1:1,000), p-p38 MAPK (no. 09-272; 1:1,000), p38 MAPK (no. 9218; 1:1,000), p-JNK (no. 4668; 1:1,000), JNK (no. 9252; $1: 1,000), \beta$-actin (no. BM0627; $1: 4,000$ ) at $4^{\circ} \mathrm{C}$ overnight. Following three washes, the membranes were incubated with goat anti-rabbit (no. ZB-2301; 1:4,000) or goat anti-mouse (no. ZB-2305; 1:4,000) IgG horseradish peroxidase-linked secondary antibodies, at room temperature for $1 \mathrm{~h}$. The bands were visualized using Immobilon Western Chemiluminescent HRP Substrate (EMD Millipore). Semi-quantification was performed using Cool Imager software (version. 4.0.1; Viagen Biotech Inc., Los Angeles, CA, USA).

Statistical analysis. All experiments were repeated at least three times. Data are presented as means \pm standard deviation. Statistical analysis was performed with SPSS software (version, 17.0; SPSS, Inc., Chicago, IL, USA). One-way analysis of variance and Student's t-test were used for comparisons. $\mathrm{P}<0.05$ was considered to indicate a statistically significant difference.

\section{Results}

Shikonin inhibits the proliferation of NB4 cells. NB4 cells were treated with increasing concentrations of shikonin for $24 \mathrm{~h}$ and viability assessed using the CCK-8 assay. There was no significant difference between the control and shikonin $0.1 \mu \mathrm{mol} / 1$ group in cell viability (Fig. 1A). However, cell viability was reduced significantly by treatment with shikonin concentrations of $0.2 \mu \mathrm{mol} / 1$ or more. The $\mathrm{IC}_{50}$ value was $0.3 \mu \mathrm{mol} / 1$. NB4 cells were also treated with $0.3 \mu \mathrm{mol} / 1$ shikonin for $0-36 \mathrm{~h}$. The CCK-8 results demonstrated that this treatment reduced cell viability significantly from $12-36 \mathrm{~h}$ (Fig. 1B). These results indicated that shikonin inhibited the viability of NB4 cells in a concentration- and time-dependent manner.

Nucleus morphological changes observed by fluorescence micsroscopy. The cell nuclei of untreated NB4 cells stained by Hoechst 33342 were round and uniform, while shikonin treatment resulted in chromatin agglutination, karyopyknosis and nuclear fragmentation (Fig. 2).

Shikonin-induced cell cycle changes. NB4 cells were treated with $0.3 \mu \mathrm{mol} / 1$ shikonin for $24 \mathrm{~h}$ and the cell cycle was detected by flow cytometry. Compared with the control group, shikonin-treated cells were arrested at the G1 phase. The percentage of cells in the G1 phase increased from 37.3 to $51.8 \%$ (Fig. 3). This result indicated that shikonin induced cell cycle arrest of NB4 cells.

Shikonin induced apoptosis of NB4 cells. The effect of $0.3 \mu \mathrm{mol} / 1$ shikonin on NB4 cell apoptosis was detected by flow cytometry at $24 \mathrm{~h}$. The percentage of apoptosis cell was increased significantly by shikonin treatment (Fig. 4).

Shikonin increased the expression levels of cleaved PARP and caspase-3. Western blotting was used to detect the apoptosis-related proteins PARP and caspase-3. The expression of cleaved PARP and caspase- 3 was increased following 
A

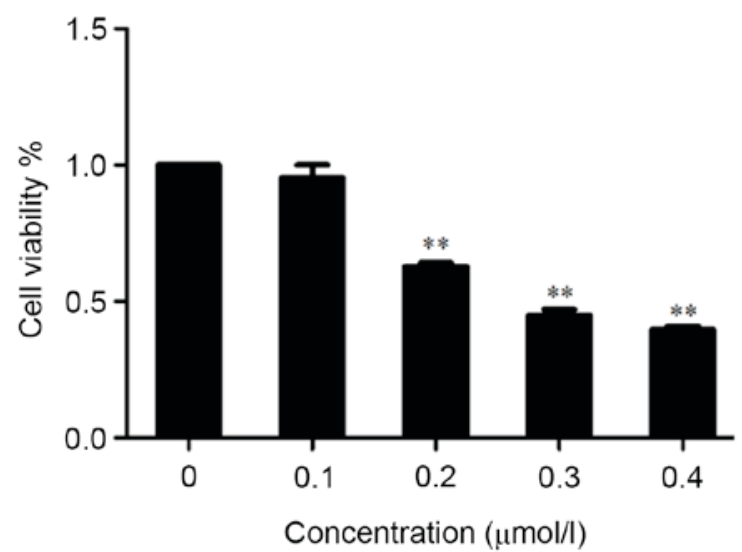

B

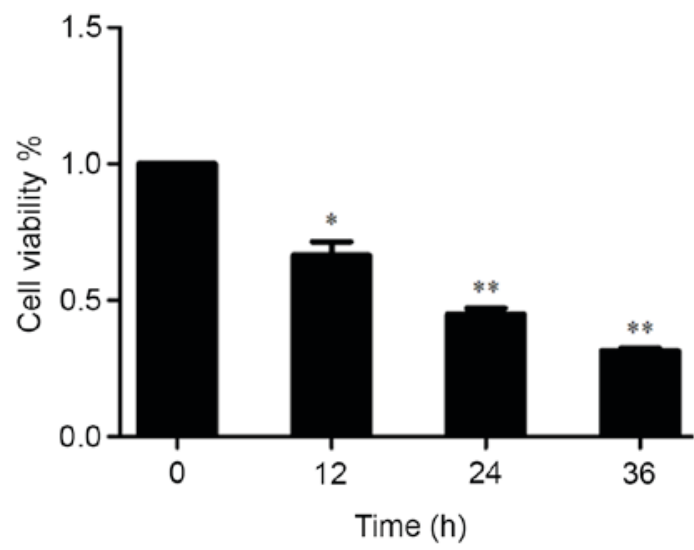

Figure 1. Treatment with shikonin inhibited human leukemia NB4 cell proliferation. (A) NB4 cells were treated with $0.1-0.4 \mu$ mol/1 shikonin for $24 \mathrm{~h} .{ }^{* * *} \mathrm{P}<0.01$ vs. $0 \mu \mathrm{mol} / 1$ shikonin. (B) NB4 cells were treated with $0.3 \mu \mathrm{mol} / 1$ shikonin for 12, 24 and $36 \mathrm{~h}$. CCK- 8 results demonstrated that shikonin inhibited NB4 cells growth in dose and time-dependent manner. ${ }^{*} \mathrm{P}<0.05,{ }^{* *} \mathrm{P}<0.01$ vs. time 0.

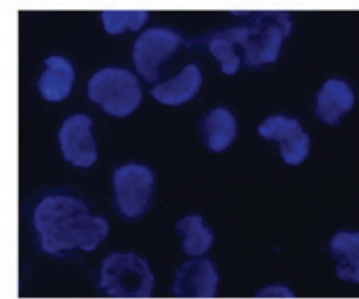

DMSO Shikonin

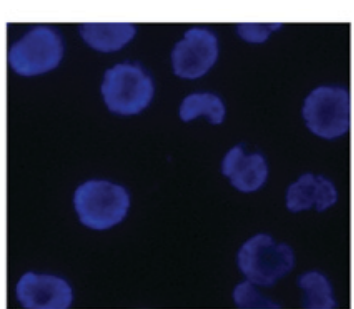

$+$
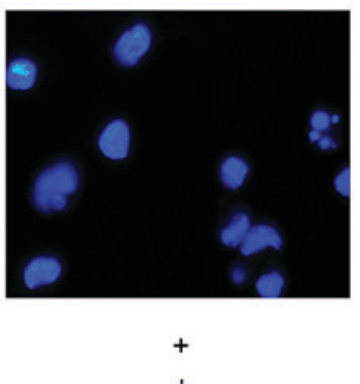

Figure 2. Effect of shikonin on nuclear morphology of NB4 cells was observed by florescence microscopy (magnification, x400). DMSO, dimethylsulfoxide.
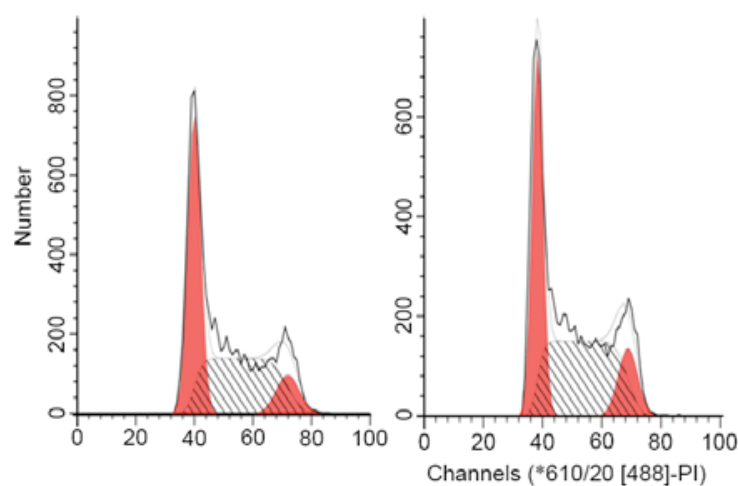

DMSO

Shikonin
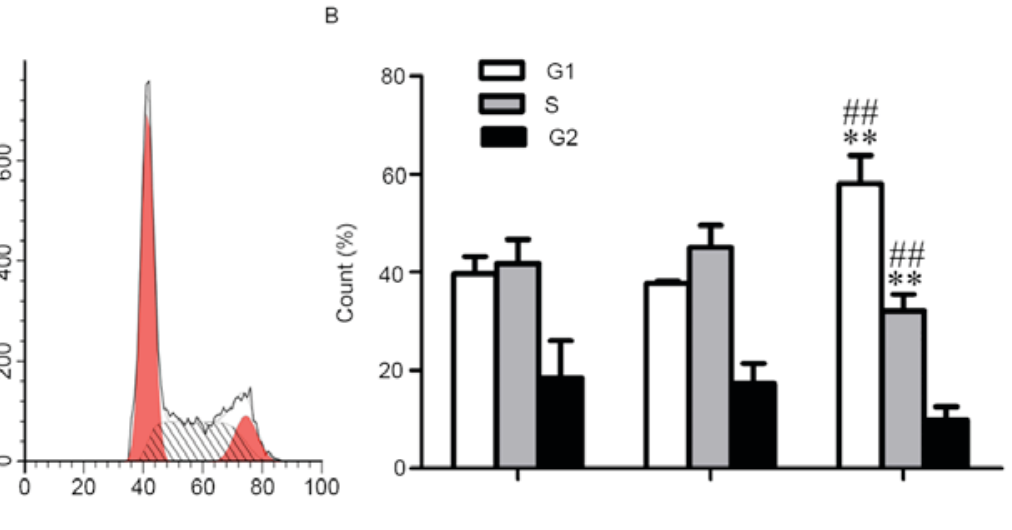

DMSO

Shikonin

Figure 3. Changes in the cell cycle distribution in NB4 cells. (A) Treatment of $0.3 \mu \mathrm{mol} / 1$ shikonin in NB4 cells for $24 \mathrm{~h}$ induced the cell cycle arrest in the G1 phase. (B) Quantification of these data. ${ }^{\#} \mathrm{P}<0.01$ vs. blank control; ${ }^{* *} \mathrm{P}<0.01$ vs. DMSO. DMSO, dimethylsulfoxide.

treatment with $0.3 \mu \mathrm{mol} / \mathrm{l}$ shikonin for $24 \mathrm{~h}$, as compared with the control group (Fig. 5), supporting the induction of apoptosis by this treatment.

Shikonin regulated MAPKs and downregulated $c-M y c$ in NB4 cells. To investigate possible mechanisms for shikonin-induced apoptosis in NB4 cells, the authors assessed the levels of MAPKs and the expression of c-Myc. Shikonin increased the phosphorylation of p38 MAPK and JNK significantly, and inhibited ERK phosphorylation (Fig. 6). However, the expression of total ERK, p38 MAPK and JNK was not affected by shikonin. Meanwhile, the 


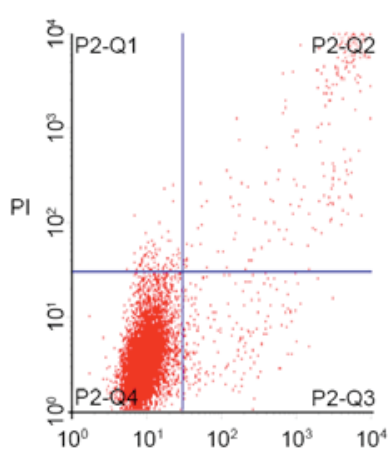

DMSO

Shikonin

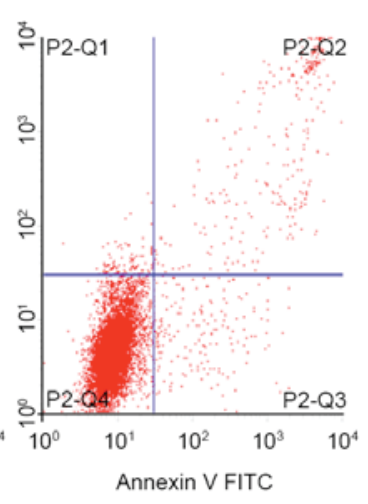

$+$
B

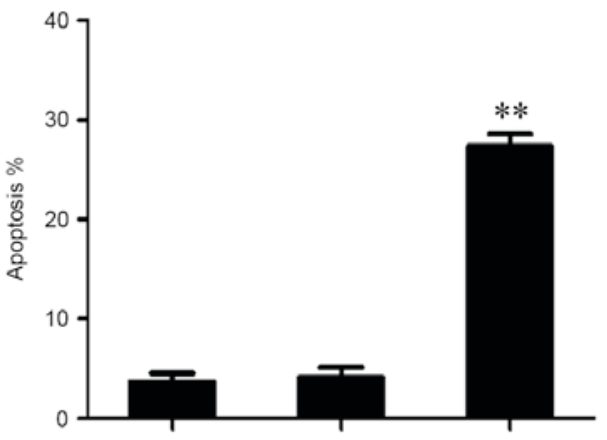

DMSO

Shikonin

Figure 4. Shikonin increased NB4 cells apoptosis. (A) NB4 cells were treated with $0.3 \mu$ mol/1 shikonin for 24 h, then cell apoptosis was detected by flow cytometry. The percentage of apoptosis cells was increased by shikonin treatment. (B) Quantification of these data. ${ }^{* *} \mathrm{P}<0.01 \mathrm{vs}$. control. DMSO, dimethylsulfoxide; FITC, fluorescein isothiocyanate.
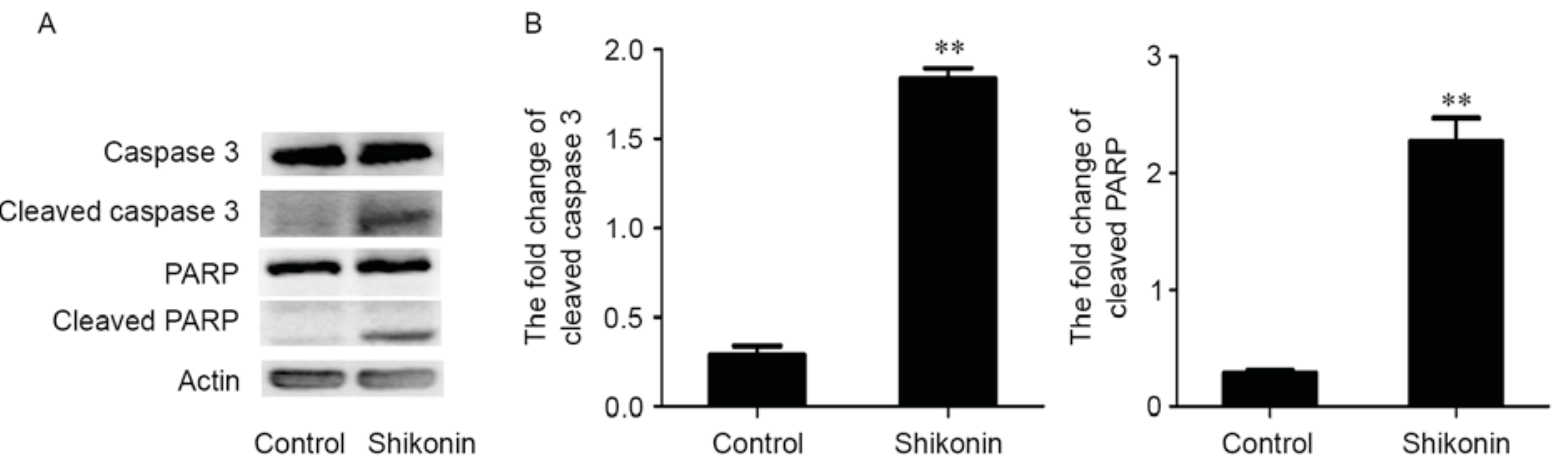

Figure 5. Effect of shikonin on the expression levels of apoptosis related proteins. (A) NB4 cells were treated with $0.3 \mu$ mol/1 shikonin for 24 h. The expression levels of cleaved caspase-3 and cleaved PARP were increased. The expression levels of caspase-3 and PARP were not changed. (B) Quantification analysis of western blotting. ${ }^{* *} \mathrm{P}<0.01$ vs. control. PARP, poly ADP-ribose polymerase.
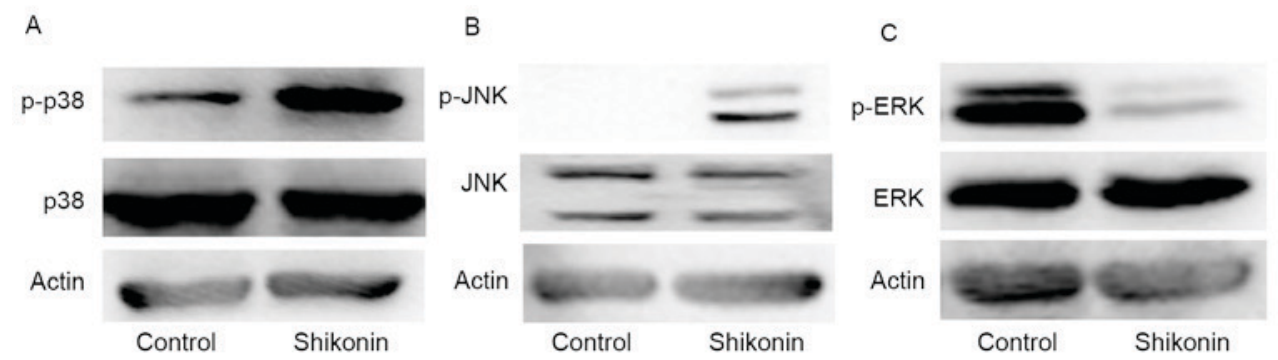

D
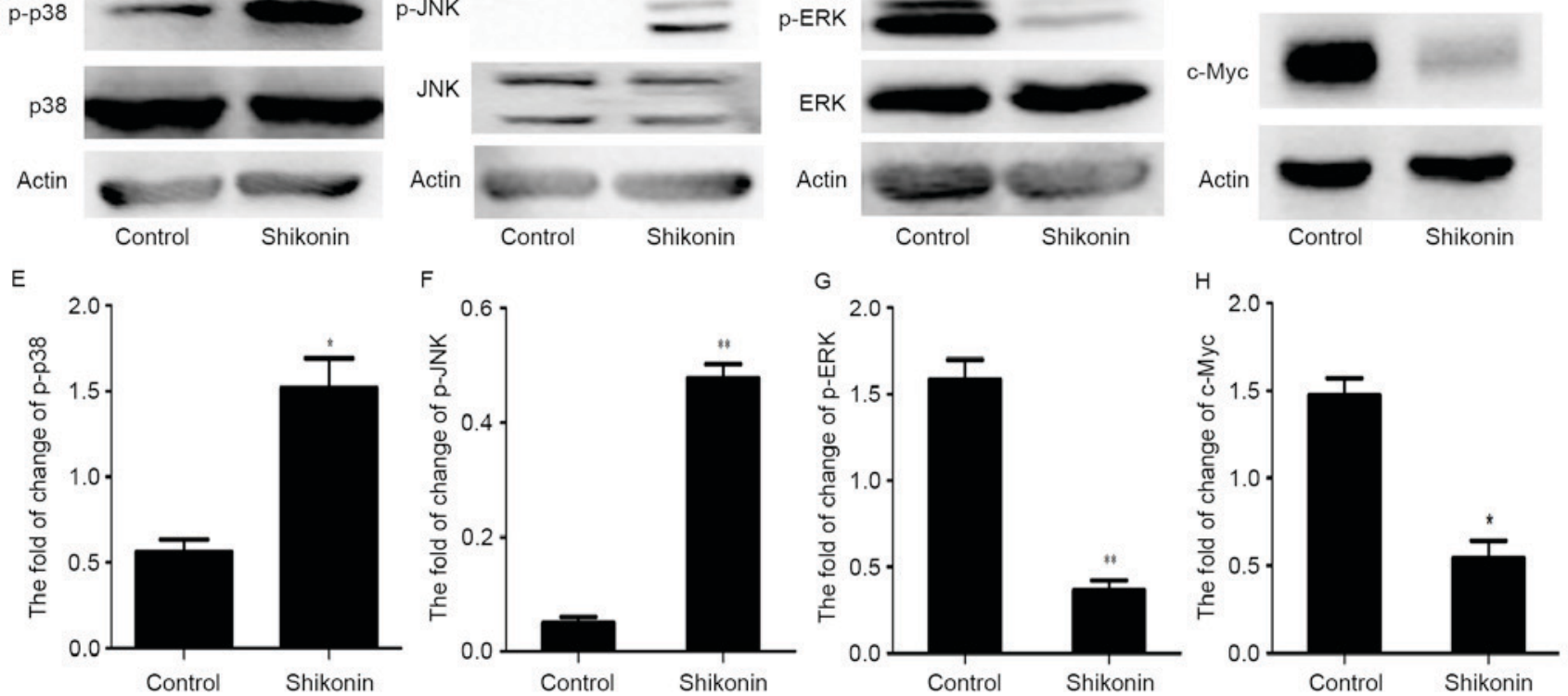

Figure 6. Effect of shikonin on the expression of mitogen-associated protein kinases and c-Myc. (A-D) NB4 cells were treated with $0.3 \mu$ mol/1 shikonin for $24 \mathrm{~h}$, and protein expression was measured by western blotting. (E-H) Quantification analysis of western blotting. * $\mathrm{P}<0.05$, $^{* *} \mathrm{P}<0.01 \mathrm{vs}$. control. JNK, Jun N-terminal kinase; ERK, extracellular signal-regulated kinase. 
expression of c-Myc was significantly decreased by treatment with shikonin.

\section{Discussion}

Shikonin, a natural product derived from the Chinese medical herb $\mathrm{Zi} \mathrm{Cao,} \mathrm{has} \mathrm{been} \mathrm{used} \mathrm{for} \mathrm{treating} \mathrm{wounds} \mathrm{and} \mathrm{burns,}$ and has anti-inflammatory and anti-viral properties $(8,9)$. Previously, evidence has indicated that shikonin exerts antitumor activity by inhibiting cell proliferation and inducing apoptosis in different tumor cell lines (10-14). However, little is known about the effects of shikonin on human leukemia NB4 cells.

In the present study, the authors firstly investigated the effects of shikonin on proliferation and apoptosis in NB4 cells. The results demonstrated that shikonin inhibited the proliferation of NB4 cells in a time- and concentration-dependent manner and induced cell cycle arrest in the G1 phase. The percentage of apoptotic cells was increased significantly following shikonin treatment. Shikonin treatment led to nucleus morphological changes such as chromatin agglutination, karyopyknosis and nuclear fragmentation. These results indicated that shikonin could inhibit the proliferation and induce apoptosis in NB4 cells. The study used NB4 cells because it is the primary cell type with the APL containing PML-RAR $\alpha$ fusion protein. In addition, the effects of shikonin on normal healthy cells were not explored because of the difficulty of culturing normal blood cells. Western blotting analyses indicated that shikonin treatment increased the cleaved caspase-3 and PARP, two apoptosis-related proteins. Furthermore, shikonin can generate reactive oxygen species and active caspases to induce apoptosis in human colorectal carcinoma cells (21). Apoptosis signal transduction involves the death receptor and mitochondrial pathways (22). In order to explore the molecular mechanism underlying the antitumor activity of shikonin, we measured its effects on cell proliferation and apoptosis signaling pathways. Studies reported that MAPK signaling pathway closely related to proliferation and apoptosis $(16,17)$. Modulation of the p38 MAPK and JNK pathways often associated with apoptosis, and the ERK pathway always related to cell survival (23-25). Western blotting examined the p38 MAPK, JNK and ERK pathways. In addition, the expression of c-Myc, an oncogene that serves an important role in cell proliferation, differentiation and apoptosis (26), and is overexpressed in many cancer cells (27), was examined. c-Myc can promote the PARP-dependent DNA repair pathway resulting in chemoresistance (28). In addition, a previous study reported that shikonin induces apoptosis by downregulating c-Myc in U937 cells (15). The current results are consistent with previous findings. Total MAPKs were unaffected by shikonin treatment. However, shikonin inhibited the expression of p-ERK and increased levels of p-p38 MAPK and p-JNK. Meanwhile, c-Myc was significantly downregulated by shikonin. These results indicated that shikonin-induced apoptosis may be through the MAPK pathway and downregulation of c-Myc in NB4 cells. However, further investigations are needed to identify how these pathways are regulated and their relationship to each other and to investigate the effects of Shikonin in animal models of APL.
In conclusion, the current study indicated that shikonin inhibited cell proliferation and induced cell cycle arrest and apoptosis in NB4 cells. These effects involved modulation of the MAPK pathway and downregulation of c-Myc. These results suggested that shikonin may be a novel agent for treating APL.

\section{Acknowledgements}

The present study was supported by the National Natural Science Foundation of China (grant no. 81171658) and the Natural Science Foundation Project of CQ CSTC (grant no. 2011BA5037).

\section{References}

1. Wang ZY and Chen Z: Acute promyelocytic leukemia: From highly fatal to highly curable. Blood 111: 2505-2515, 2008.

2. Albano F, Zagaria A, Anelli L, Coccaro N, Tota G, Brunetti C, Minervini CF, Impera L, Minervini A, Cellamare A, et al: Absolute quantification of the pretreatment PML-RARA transcript defines the relapse risk in acute promyelocytic leukemia. Oncotarget 6: 13269-13277, 2015.

3. Powell BL: Arsenic trioxide in acute promyelocytic leukemia: Potion not poison. Expert Rev Anticancer Ther 11: 1317-1319, 2011.

4. Zhang K, Li J, Meng W, Xing H and Yang Y: Tanshinone IIA inhibits acute promyelocytic leukemia cell proliferation and induces their apoptosis in vivo. Blood Cells Mol Dis 56: 46-52, 2016.

5. Li L, Song H, Zhong L, Yang R, Yang XQ, Jiang KL and Liu BZ: Lithium chloride promotes apoptosis in human leukemia NB4 cells by inhibiting glycogen synthase kinase-3 beta. Int J Med Sci 12: 805-810, 2015.

6. Iland HJ, Bradstock K, Supple SG, Catalano A, Collins M, Hertzberg M, Browett P, Grigg A, Firkin F, Hugman A, et al: All-trans-retinoic acid, idarubicin and IV arsenic trioxide as initial therapy in acute promyelocytic leukemia (APML4). Blood 120: 1570-1580, 2012.

7. Ghavamzadeh A, Alimoghaddam K, Rostami S, Ghaffari SH Jahani M, Iravani M, Mousavi SA, Bahar B and Jalili M: Phase II study of single-agent arsenic trioxide for the front-line therapy of acute promyelocytic leukemia. J Clin Oncol 29: 2753-2757, 2011.

8. Papathanasiou K, Papageorgiou C, Panidis D and Mantalenakis S: Our experience in laparoscopic diagnosis and management in women with chronic pelvic pain. Clin Exp Obstet Gynecol 26: 190-192, 1999.

9. Chen X, Yang L, Oppenheim JJ and Howard MZ: Cellular pharmacology studies of shikonin derivatives. Phytother Res 16: 199-209, 2002.

10. Gaddipati JP, Mani H, Shefali, Raj K, Mathad VT, Bhaduri AP and Maheshwari RK: Inhibition of growth and regulation of IGFs and VEGF in human prostate cancer cell lines by shikonin analogue 93/637 (SA). Anticancer Res 20: 2547-2552, 2000.

11. Min R, Tong J, Wenjun Y, Wenhu D, Xiaojian Z, Jiacai H, Jian Z, Wantao $\mathrm{C}$ and Chenping Z: Growth inhibition and induction of apoptosis in human oral squamous cell carcinoma Tca-8113 cell lines by shikonin was partly through the inactivation of NF-kappaB Pathway. Phytother Res 22: 407-415, 2008.

12. Mao X, Yu CR, Li WH and Li WX: Induction of apoptosis by shikonin through a ROS/JNK mediated process in Bcr/Abl-positive chronic myelogenous leukemia (CML) cells. Cell Res 18: 879-888, 2008.

13. Gong K and Li W: Shikonin, a Chinese plant-derived naphthoquinone, induces apoptosis in hepatocellular carcinoma cells through reactive oxygen species: A potential new treatment for hepatocellular carcinoma. Free Radic Biol Med 51: 2259-2271, 2011.

14. Yang Q, Ji M, Guan H, Shi B and Hou P: Shikonin inhibits thyroid cancer cell growth and invasiveness through targeting major signaling pathways. J Clin Endocrinol Metab 98: E1909-E1917, 2013.

15. Zhao Q, Assimopoulou AN, Klauck SM, Damianakos H, Chinou I, Kretschmer N, Rios JL, Papageorgiou VP, Bauer R and Efferth T: Inhibition of c-MYC with involvement of ERK/JNK/MAPK and AKT pathways as a novel mechanism for shikonin and its derivatives in killing leukemia cells. Oncotarget 6: 38934-38951, 2015. 
16. Zhang W and Liu HT: MAPK signal pathways in the regulation of cell proliferation in mammalian cells. Cell Res 12: 9-18, 2002.

17. Cobb MH: MAP kinase pathways. Prog Biophys Mol Biol 71: 479-500, 1999

18. Dent P and Grant S: Pharmacologic interruption of the mitogen-activated extracellularregulated kinase/mitogen-activated protein kinase signal transduction pathway: Potential role in promoting. Clin Cancer Res 7: 775-783, 2001.

19. Fan $M$ and Chambers TC: Role of mitogen-activated protein kinases in the response of tumor cells to chemotherapy. Drug Resist Updat 4: 253-267, 2001.

20. Dent $P$ and Grant S: Pharmacologic interruption of the mitogen-activated extracellular-regulated kinase/mitogen-activated protein kinase signal transduction pathway: Potential role in promoting cytotoxic drug action. Clin Cancer Res 7: 775-783, 2001.

21. Hsu PC, Huang YT, Tsai ML, Wang YJ, Lin JK and Pan MH: Induction of apoptosis by shikonin through coordinative modulation of the Bcl-2 family, p27, and p53, release of cytochrome c, and sequential activation of caspases in human colorectal carcinoma cells. J Agric Food Chem 52: 6330-6337, 2004.

22. van Engeland M, Ramaekers FC, Schutte B and Reutelingsperger CP: A novel assay to measure loss of plasma membrane asymmetry during apoptosis of adherent cells in culture. Cytometry 24: 131-139, 1996.
23. Moon DO, Kim MO, Choi YH, Kim ND, Chang JH and Kim GY: Bcl-2 overexpression attenuates SP600125-induced apoptosis in human leukemia U937 cells. Cancer Lett 264: 316-325, 2008.

24. Cross TG, Scheel-Toellner D, Henriquez NV, Deacon E, Salmon M and Lord JM: Serine/threonine protein kinases and apoptosis. Exp Cell Res 256: 34-41, 2000.

25. Park HS, Hwang HJ, Kim GY, Cha HJ, Kim WJ, Kim ND, Yoo $\mathrm{YH}$ and Choi YH: Induction of apoptosis by fucoidan in human leukemia U937 cells through activation of p38 MAPK and modulation of Bcl-2 family. Mar Drugs 11: 2347-2364, 2013.

26. Pan XN, Chen JJ, Wang LX, Xiao RZ, Liu LL, Fang ZG, Liu Q, Long ZJ and Lin DJ: Inhibition of c-Myc overcomes cytotoxic drug resistance in acute myeloid leukemia cells by promoting differentiation. PLoS One 9: e105381, 2014

27. Lu JJ, Meng LH, Shankavaram UT, Zhu CH, Tong LJ, Chen G, Lin LP, Weinstein JN and Ding J: Dihydroartemisinin accelerates c-MYC oncoprotein degradation and induces apoptosis in c-MYC-overexpressing tumor cells. Biochem Pharmacol 80: 22-30, 2010.

28. Ganesan S: MYC, PARP1, and chemoresistance: BIN there, done that? Sci Signal 4: pe15, 2011. 